\title{
RNA Sequences from Cambridge
}

YET another sequence of nucleotide bases in an RNA molecule has been worked out by members of Dr F. Sanger's group at the MRC Laboratory for Molecular Biology (see page 234). What Dube, Marcker, Clark and Cory have done is to determine the nucleotide sequence of $\mathrm{N}$-formyl-methionyl-transfer RNA (F-met$t \mathrm{RNA}_{\mathrm{F}}$ ) from $E$. coli. The determination of the nucleotide sequences of ribosomal, transfer and messenger RNAs and of DNA is a prerequisite for the correlation of the structure of these molecules and their function. This is one of the outstanding, and certainly one of the most tedious, of the remaining problems in molecular biology, and the decision at Cambridge to concentrate on sequence analysis of nucleic acids is clearly paying handsome dividends.

Having bcen the first to analyse the amino-acid sequence of a protein, it is hardly surprising that Dr Sanger and his colleagues have developed such successful methods for the separation and analysis of oligonucleotides. Naturally enough, they have concentrated on small RNA molecules-the nucleic acid equivalents of insulin. In 1967, Brownlee, Sanger and Barrell reported the sequence of $5 S$ ribosomal RNA (Nature, 215, 735; 1967). Recently (Nature, 21\%, 109; 1968) Goodman, Abelson, Landy, Brenner and Smith, using Sanger's methods, described how they determined the sequences of tyrosine $t$ RNA and the amber suppressor $s u_{\text {III }}^{+}$tyrosine $t$ RNA of $E$. coli and proved that mutation to amber suppression results from a single base change in the anticodon triplet of one of the tyrosine $t$ RNAs. The anticodon of the tyrosine $t$ RNA is GUA and the $s u_{\mathrm{III}}^{+}$anticodon is CUA. Apart from this difference, the sequences of the rest of the two molecules are identical. This result formally identifies the anticodon loop of the transfer RNAs. The function of the other parts of the molecule can now be studied with mutants. Apparent revertants of the $s u_{I_{I I}^{+}}^{+}$gene to $s u^{-}$, which are no longer amber suppressors, may either have CUA as anticodon, in which case they are true revertants, or they may have additional mutations in other sites which destroy the function of the molecule. Revertants to $s u^{-}$map in various regions of the $t \mathrm{RNA}$ gene, and analyses of their biological function and nucleotide sequences, which are already in progress, should give clues about the role of the rest of the $t$ RNA molecule apart from the anticodon loop.

Although this group has worked on suppressor $t$ RNAs, Dube et al. have concentrated on another very interesting species of $t \mathrm{RNA}$, the $\mathrm{N}$-formyl-methionyl$t$ RNA which initiates protein synthesis in $E$. coli. How does F-met-tRNA - differ from the methionine $t$ RNA, which has similar properties to all the other species of $t$ RNA and which inserts methionine at internal positions during protein synthesis? To try to answer this, Dube et al. embarked on a comparison of the nucleotide sequences of met-tRNA and F-met- $t \mathrm{RNA}_{\mathrm{F}}$.
Although the sequence of met-tRNA is not completed and the comparison cannot yet be made, the sequence of F-met- $t$ RNAF shows several interesting features which may be related to its role in initiation. The anticodon is CAU. The third base of this anticodon, the U, can, according to Crick's hypothesis, "wobble" and pair with either A or G; it has also been firmly established that two codons, AUG and GUG, can be read as initiators. Thus "wobbln" at initiation involves the first base of the codon and the third base of the anticodon. All other cases of wobble involve the third base of the codon and the first base of the anticodon (see Nature, 216,$431 ; 1967$ ) and it is possible that the unique first base-pair wobbling of $\mathrm{F}$-met$t \mathrm{RNA}_{\mathrm{F}}$ depends on the base immediately following the anticodon being unmodified.

Apart from F-met $t \mathrm{RNA}_{\mathrm{F}}$ and yeast valine $t \mathrm{RNA}$, the base immediately following the anticodon in all the species of $t$ RNA of which the sequences have so far been determined is either a modified $A$ or a rare base such as methyl inosine. (In the case of yeast valine $t$ RNA, the last base of the anticodon is C, which according to Crick's hypothesis would never be able to wobble.) Thus it may be that there is a rule that third base wobble, a property of non-initiating $t$ RNA, depends on the presence of a modified base immediately after the anticodon, and if the base in this position is unmodified, wobble occurs in the first base position. It will be interesting to see whether met-tRNA has modified $\mathrm{A}$ or a rare base where F-met- $t \mathrm{RNA}_{\mathrm{F}}$ has unmodified A.

The nucleotide sequence of F-met- $t$ RNA has two other unusual features. In the right-hand loop of the cloverleaf there is an unusual sequence and the $5^{\prime}, 3^{\prime}$ base pairing is unusual. The $5^{\prime}$ terminal base $\mathrm{C}$ does not pair with the fifth base in from the $3^{\prime}$ end, which is A. In all other transfer RNAs, the bases at these positions are paired. Some of the other properties of F-met- $t$ RNA , such as the specificity of formylation, may depend on these sequences, and a knowledge of the tertiary structure of the molecule would provide the answers. That is why experiments to test the properties of large fragments of the molecule are being carried out.

\section{Substitute for Scots Pine}

LODGEPOLE pine, which was introduced into Britain from America in 1853, but only grown extensively in the past twenty years, is likely to be a satisfactory alternative to Scots pine for roofing, flooring and similar purposes. This point is made in a booklet, Lodgepole Pine, produced by the Forest Products Research Laboratory (HMSO, 5s.).

Pinus contorta, lodgepole pine, grows along the western coast of North America from Alaska to California, inland along the Sierra Cascades from the Canadian border to southern California, and further inland along 